\title{
Siu-Lan Tan, Peter Pfordresher, \& Rom Harré, Psychology of music: From sound to significance. Hove, UK: Psychology Press, 2010. ISBN: 978-1-84169-868-7 (hardcover)
}

The field of Music Psychology has received formative influences from many domains, thus it is no mean feat to create a representative survey of the literature. The authors have met the challenge, achieving a detailed and useful introduction to the field with this text. They have covered a range of perspectives, including philosophy, modelling, cognitive psychology, neuroscience, acoustics, education, social psychology, musicology, and music theory. They even venture into unusual but interesting applied areas such as architectural acoustics and gender bias. The authors strike a good balance between giving enough detail to understand, but not so much as to overwhelm. However, in the few spots where those new to the field may be left scratching their heads, a glossary would have been a helpful addition. In addition, although it is tricky to provide auditory examples in textbooks, I felt that for some sections (e.g., properties of sound, cross-cultural music comparisons), auditory examples could significantly improve the reader's understanding.

Chapter 1 lays out the progression of the book, which begins with the physical basis for musical sound, proceeds to perception and cognition, then to development of musical experience, and finally to meaning and emotion. The first chapter helpfully flags the chapters that may be skipped by the more experienced reader, and two chapters that may be somewhat difficult for those without a background in music theory. The intended audience includes people who may become music psychologists, people looking for more advanced foundations, or casual readers exploring a new field. The latter may find the amount of information in some sections challenging, but there is an abundance of illustrations and observations that bring out the everyday relevance of the material.

Chapters $2-4$ cover the basics of sound and our perception of it. Chapter 2 considers basic musical acoustics and, along with Chapter 3 , was one place that auditory examples would have helped to demonstrate the material. The chapter finishes with a rather unusual topic, architectural acoustics, which is lucidly presented and an extremely enjoyable read. In Chapter 3 we are introduced to the fundamentals of sound and neurophysiology of the auditory system. The terminology surrounding the concepts of pitch, timbre, amplitude, and duration is explained well, with the possible exception of critical bandwidth which I found a bit confusing at first. The physiology and structure of the auditory system is intelligibly laid out and well-illustrated. In Chapter 4 the methods of cognitive neuroscience and anatomical brain terminology are covered. The narrative here was less clear than in the rest of the book, as the methods are described largely in isolation from empirical studies, then illustrated by investigations in a few areas of neuroscience and music (mainly language and pitch). To me, this made it difficult to understand how neuroscience methods could contribute to our understanding of cognitive and theoretical aspects of music processing. For example, what does a change in the amplitude of an event-related potential mean? We are told that ERPs 'would be of interest' to neuroscientists, but not why, or why non-neuroscientists might also be interested. This reflects something of a general issue in the field: even some neuroscientists are guilty of failure to communicate their relevance of their findings to the cognitive domain, but I would have liked to see the relationship between neuroscience and cognition more clearly spelled out in the discussion of the methods. The subsequent coverage of neuroscientific studies goes some way to relating the findings gained through neuroscientific methods to cognitive theories, but reads as a bit of a 'taster' of neuroscience findings. However, the explanation of the methods themselves is admirably clear, and several good resources for further neuroscience reading are given at the end of the chapter.

Chapters 5- 7 cover the perception and cognition of music. Pitch and melody are reviewed first, with comprehensive coverage of the important concepts in both perceptual organization of and memory for pitch-related constructs. The description of Gestalt grouping principles is particularly well-crafted, as is the explanation of musical key involving dissonance avoidance within a critical bandwidth. Research on rhythm and time has expanded rapidly in the last decade, and Chapter 6 gives a fairly up-to-date account. In the neuroscience section, I would have made a different choice about highlighting Sakai et al.'s (1999) study of rhythm, as the statistical methods prevent one from reliably interpreting the reported brain activation differences, but in general the key concepts are well-represented. Chapter 7 is about theories of musical structure, and does an excellent job of describing Lerdahl and Jackendoff's Generative Theory of Tonal Music (Lerdahl \& Jackendoff, 1983) and Narmour's implication-realization model (Narmour, 1990, 1992). However, I think all but a dedicated few with music theory background will find it a bit abstract, and 
some comparisons with statistical learning approaches (Pearce, Ruiz, Kapasi, Wiggins, \& Bhattacharya, 2010; Saffran, Johnson, Aslin, \& Newport, 1999) might have provided an interesting contrast.

After covering the foundations and basic perceptual processes, the book steps up a gear; I found the last half to be a genuine pleasure to read. The authors link the topics (development, music performance, social psychology, and emotion) closely to our real-world experiences of music, and delve deeply into areas sometimes glossed over in other texts, such as the fetal soundscape. Chapter 8 describes the development of prenatal and infant musical perception, covering both old and new research. The sections on associations between maternal heartbeat and a newborn's responses, as well as the benefits of musical exposure for babies are other examples of practical grounding that will pique the interest of a wide range of readers. Chapter 9 moves on to infants and young children, integrating influential theories of early musical education (Orff, Dalcroze, and Kodaly) with empirical studies of children's musical behaviour. The indepth coverage of the spontaneous musical activity exhibited by children is particularly illuminating, and emphasizes that musical activity is about much more than formal training (a point that is also driven home in the social psychology chapter). Chapter 10 covers the nature/nurture debate, and studies of expertise and its relationship to deliberate practice, including the popular " 10,000 hours" rule. This is integrated with empirical studies of music performance that investigate different practice strategies (distributed versus massed practice, part versus whole practice approaches, and mental imagery). The chapter ends with coverage of brain plasticity in musicians and the effects of training on perception. Here I would have liked to see Schneider et al.'s work (Schneider, et al., 2002; Schneider, Sluming, Roberts, Bleeck, \& Rupp, 2005), which goes beyond many plasticity studies in demonstrating a functional link between the size of brain structures (Heschl's gyrus) and perceptual abilities and preferences. In Chapter 11, the psychology of music performance is considered, from working memory, expressive timing, and tuning through to singing ability in the general population.

The final section is entitled 'the meaning and significance of music'. It opens with Chapter 12, 'the social psychology of music'. The material here will be interesting to a variety of readers, as it deals with topics such as our social constructs about behaviour at musical events and the influence of music on consumer behaviour. A novel inclusion is the section on music and gender that considers stereotypes associated with different musical instruments and genres, in addition to the self-perceptions of male and female musicians. Chapter 13 is about the question of meaning in music. Major theories of the relationship between music and emotion are given in-depth treatment, and although a bit drier than surrounding chapters, the theories are coherently conveyed. Chapter 14 covers emotion, starting with expression and communication of emotion in music and music performance, moving onto physiological and neural responses, and finishing with engaging empirical reports of how movie scores affect emotional judgements. The final chapter is on culture and music, and includes discussion of music in nonhuman animals, musical universals, and specific tonal and rhythmic differences in music across cultures. The literature about the cultural specificity of musical constructs is interesting, but I suspect this is another section that may have benefited from musical examples. It is difficult to communicate gamelan instrument tuning or the rhythmic patterns in Indian ragas through text alone (although the authors do a reasonable job of it).

Overall I found the book comprehensive and cogently presented. Throughout the text, the style is engaging and personal. Anecdotes and personal insights are given by the different authors, and researchers are introduced by first and last name. Throughout, a number of illustrative examples are drawn from daily life. The detail may, at times, be a bit for a newcomer to grapple with, but anyone who masters the content of this text can certainly consider themselves proficient in the topic of music psychology.

Jessica A. Grahn

Centre for Brain and Mind Department of Psychology University of Western Ontario London, Ontario

Email: jgrahn@uwo.ca 


\section{References}

Lerdahl, F., \& Jackendoff, R. (1983). A Generative Theory of Tonal Music. Cambridge, MA: MIT Press.

Narmour, E. (1990). The Analysis and Cognition of Basic Melodic Structures: The Implication-Realization Model. Chicago: University of Chicago Press.

Narmour, E. (1992). The Analysis and Cognition of Melodic Complexity: The Implication-Realization Model. Chicago: University of Chicago Press.

Pearce, M. T., Ruiz, M. H., Kapasi, S., Wiggins, G. A., \& Bhattacharya, J. (2010). Unsupervised statistical learning underpins computational, behavioural, and neural manifestations of musical expectation. NeuroImage, Vol. 50, No. 1, pp. 302-313.

Saffran, J. R., Johnson, E. K., Aslin, R. N., \& Newport, E. L. (1999). Statistical learning of tone sequences by human infants and adults. Cognition, Vol. 70, No. 1, pp. 27-52.

Sakai, K., Hikosaka, O., Miyauchi, S., Takino, R., Tamada, T., Iwata, N. K., et al. (1999). Neural representation of a rhythm depends on its interval ratio. Journal of Neuroscience, Vol. 19, No. 22, pp. 10074-10081.

Schneider, P., Scherg, M., Dosch, H. G., Specht, H. J., Gutschalk, A., \& Rupp, A. (2002). Morphology of Heschl's gyrus reflects enhanced activation in the auditory cortex of musicians. Nature Neuroscience, Vol. 5, pp. 688-694.

Schneider, P., Sluming, V., Roberts, N., Bleeck, S., \& Rupp, A. (2005). Structural, functional, and perceptual differences in Heschl's gyrus and musical instrument preference. Annals of the New York Academy of Sciences, Vol. 1060, pp. 387-394. 